University of New Hampshire

University of New Hampshire Scholars' Repository

10-1987

\title{
On-Line Sea Beam Acoustic Imaging
}

Christian de Moustier

University of California - San Diego

F V. Pavlicek

University of California - San Diego

Follow this and additional works at: https://scholars.unh.edu/ccom

Part of the Computer Sciences Commons, and the Oceanography and Atmospheric Sciences and Meteorology Commons

\section{Recommended Citation}

de Moustier, Christian and Pavlicek, F V., "On-Line Sea Beam Acoustic Imaging" (1987). IEEE Oceans. 763. https://scholars.unh.edu/ccom/763

This Conference Proceeding is brought to you for free and open access by the Center for Coastal and Ocean Mapping at University of New Hampshire Scholars' Repository. It has been accepted for inclusion in Center for Coastal and Ocean Mapping by an authorized administrator of University of New Hampshire Scholars' Repository. For more information, please contact Scholarly.Communication@unh.edu. 


\title{
ON-LINE SEA BEAM ACOUSTIC IMAGING
}

\author{
C. de Moustier and F.V. Pavlicek
}

Marine Physical Laboratory

Scripps Institution of Oceanography

La Jolla, CA 92093, U.S.A.

\begin{abstract}
This paper describes a system designed and built at the Marine Physical Laboratory of the Scripps Institution of Oceanography to produce acoustic images of the seafloor on-line with a Sea Beam multibeam echo-sounder. This system uses a stand alone interface between the Sea Beam system and a grey-scale line-scan recorder. The interface is built around a Motorola 68000 microprocessor and has digitizing capabilities. It digitizes the detected echo signals from each of the 16 preformed beams inside the Sea Beam echo processor as well as the roll information given by the ship's vertical reference. The acoustic data are then roll compensated and combined into a port and a starboard time series. These time series are eventually output in digital format to a line-scan recorder which produces the grey scale acoustic image. Results are discussed for Sea Beam acoustic images of the seafloor and of the Deep Scattering layers.
\end{abstract}

\section{INTRODUCTION}

As part of a program to investigate seafloor acoustic backscatter measured with a Sea Beam multibeam echo-sounder, the Marine Physical Laboratory (MPL) of the Scripps Institution of Oceanography built a parallel acoustic data acquisition system using a DEC LSI-11/73 minicomputer $(1,2)$ to preserve the echo signals received by the Sea Beam system which has no internal provisions to do so. The Sea Beam system is primarily a high-resolution bathymetric survey tool which transmits a $7 \mathrm{~ms}$ pulse of $12.158 \mathrm{kHz}$ over a fanshaped beam $\left(22 / 3^{\circ}\right.$ fore-aft by $54^{\circ}$ athwartships) stabilized in pitch, and which receives bottom echoes on 16 adjacent preformed beams (each $20^{\circ}$ fore-aft by $22 / 3^{\circ}$ athwartships). It processes the echoes and outputs a contour chart of a swath of seafloor with a width roughly equal to $3 / 4$ of the water depth below the ship. For detailed descriptions of the Sea Beam system the reader is referred to the works by Renard and Allenou (3), Farr (4) and de Moustier and Kleinrock (5)

While analyzing bottom echo signals received by the Sea Beam system and recorded with this parallel data acquistion system, we found that in spite of the multibeam geometry, the equivalent of a side-scan sonar image of the seafloor could be obtained by combining beams on either side of vertical (6). Such an acoustic image of the seafloor yields qualitative information about the texture of the bottom and complements the high-resolution bathymetry normally derived from the Sea Beam system. Because this textural information is a potentially important clue for geomorphological interpretations, it would be very valuable to the investigator in real time during the course of a survey. For this reason, we decided to build an on-line acoustic imaging system which would give immediate access to the data as opposed to recording them on tape and producing grey-scale displays in a post-processing operation.

In the following, we describe this on-line Sea Beam acoustic imaging system which consists of a "smart" interface, built around a Motorola 68000 microprocessor, between the Sea Beam system and a line-scan recorder. We first give an overview of the general system configuration with the various peripherals involved; we then describe the microprocessor-controlled unit that orchestrates the acoustic imaging scheme, and we presents some data samples.

\section{GENERAL SYSTEM ARCHITECTURE}

In this section, we briefly describe the general configuration of the acoustic imaging system and its links to the Sea Beam echosounder.

As seen in Figure 1, the major components of the system consist of a microprocessor-controlled unit (stippled area) interfaced to a Sea Beam system, a set of peripherals including a CRT console for operator interactions and a line-scan recorder for data output, and a link to a host processor for software down-loading. We use the DEC LSI-11/73 minicomputer of our acoustic data acquisition system mentioned above as the host processor. The line-scan recorder is a 4-bit grey scale Raytheon LSR-1807M dry paper recorder with digital input and 4 kbytes of memory.

Three types of signals are taken from the Sea Beam system; clock signals for time reference and synchronization, roll signals for vertical reference and acoustic signals from the 16 beams. To minimize interferences with the Sea Beam system, the acoustic signals and the clock signals are tapped by high-impedance buffer amplifiers inside the Sea Beam system, and are transferred differentially to the interface. The roll signals are transformer coupled.

The microprocessor-controlled unit consists of 3 boards linked by a 16-bit data bus. The heart of the unit is the processor board which includes a 68000 processor, 16 kbytes of EPROM holding monitor software and 20 kbytes of RAM for monitor management functions. This board also has serial ports that are used for RS-232 links to the operator's console and to the host processor. The second board is a memory expansion board configured to hold up to 256 kbytes of memory. In its current configuration, 192 kbytes of RAM are installed on this board. Provision has been made to install up to 64 kbytes of EPROM's in order to make a completely stand alone system. The third board is the interface board which services inputs from the Sea Beam system and from the unit's front panel, and outputs to the line-scan recorder. The unit's front panel features a set of 8 switches for operator interaction (setting of initial values or modifying program constants) and a two-digit LED display for performance monitoring. 


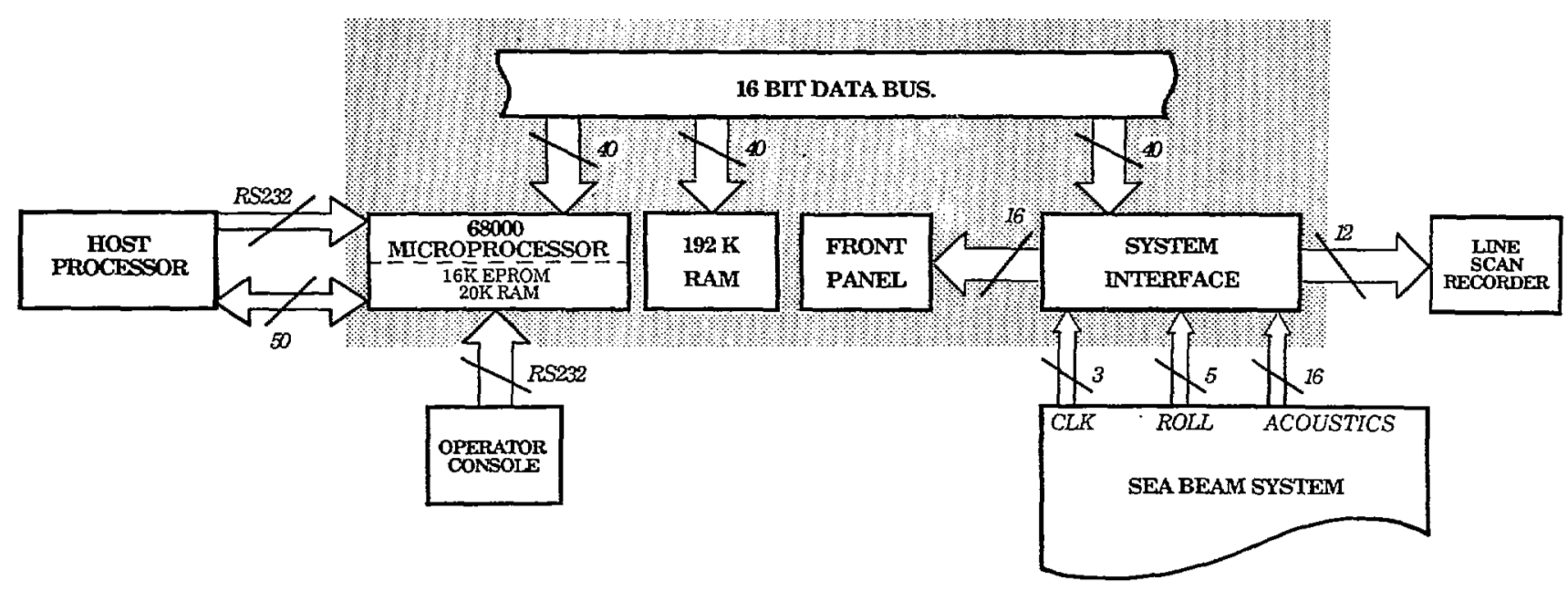

Figure 1: General system block diagram, the stippled area delimits the microprocessor system.

The processor board and the memory expansion board are off-theshelf units and we will not discuss them further in this paper. On the other hand, we have configured the interface board specifically for this system and we shall describe it in more details in the following section.

\section{INPUT-OUPUT INTERFACE}

As indicated in the previous section, the main function of the input-output interface board is to service inputs from the Sea Beam system and the unit's front panel as well as outputs to the recorder.

Figure 2 illustrates the signal paths from the Sea Beam system to the microprocessor through the interface. At the center of this interface is a data control and timing unit which gets its inputs from the sonar key pulse and the Sea Beam system's $12.158 \mathrm{kHz}$ reference frequency. The sonar key pulse indicates the onset of a transmission cycle in the Sea Beam system and it serves here as the time zero reference. The $12.158 \mathrm{kHz}$ reference frequency produced by the Sea Beam system's transmit signal generator is fed into a phase-lock loop whose output is divided down and used as the digitizing clock on the interface board. The roll signals come in synchro format (3 phases $120^{\circ}$ apart and $400 \mathrm{~Hz}$ reference) from the ship's vertical reference and are fed to a synchro-to-digital (S/D) converter. The outputs of the S/D converter are held in Dtype registers which interface directly with the system bus so that the processor can access the roll data directly at a dedicated memory address. The 16 acoustic channels corresponding to the 16 Sea Beam preformed beams are input differentially into a 16channel analog multiplexer whose common output is held in a sample and hold amplifier interfaced to a 12-bit analog-to-digital (A/D) converter. As mentioned above, the $\mathrm{A} / \mathrm{D}$ converter is clocked by a timing signal which is an integer sub-multiple of the $12.158 \mathrm{kHz}$ reference frequency. The multiplexer is also synchronously controlled to change channels between each $A / D$ digitizing cycles. In the present configuration, the sampling rate is approximately 500 $\mathrm{Hz}$ per channel. The digitized data is stored in a 512 -word First-in First-out (FIFO) memory which is directly accessible to the processor through the system bus.

Inputs from the switches on the unit's front panel are directed to the system bus by way of bus drivers whenever the microprocessor reads data from the memory address assigned to the front panel. Likewise, bus access to the LED display on the front panel is done directly through D-type registers when the microprocessor writes to the front panel's memory address.
Outputs to the line-scan recorder are interrupt driven. The recorder issues a "time zero" interrupt to the processor when the stylus-reaches the left hand edge of the paper. When the processor is ready to service this interrupt, it writes four bits of data (a nibble) to a D-type register at the address of the recorder parallel port. The microprocessor sends 4096 such nibbles to this address and a separate circuit strobes the data into the recorder buffer. Note that this sequence of events is specific to a Raytheon LSR-1807M recorder; provisions have been made on the interface board to accomodate EPC-type recoders which interrupt the processor for each transmission of a 4-bit pixel in addition to the aforementioned time zero interrupt.

\section{Timing Sequence for Data Acquisition}

The various input and output operations outlined above are managed by the data control and timing unit according to a prescribed sequence for data acquisition. The timing diagram shown in Figure 3 illustrates the sequence of events taking place between two sonar key pulses (time zero) during data acquisition. The sonar key pulse triggers a decrementing counter previously loaded with a time delay proportional to the time necessary for sound to reach the seafloor and return to the ship. When a program is first started, this depth dependent time delay must be set from the front panel switches according to the current water depth below the ship. - From then on, the program tracks the bottom and updates the delay timer automatically.

When the timer reaches zero, it starts the $A / D$ and the S/D conversions. As indicated above, the output of the $\mathrm{A} / \mathrm{D}$ converter is held in a 512-word FIFO memory. The FIFO sends an interrupt to the processor on the half-full flag ( 256 words) and the processor must read data off the FIFO into memory in order to clear the interrupt. A/D conversions and FIFO interrupts continue until the specified number of samples has been digitized for each channel and stored into memory. For the S/D data, the processor need only read the data off the bus and interleave the roll samples into memory with every 256-word interrupt sequence.

The next set of operations includes data processing and displays. The data processing is mainly concerned with automatic bottom tracking, roll compensation and formatting of the digitized acoustic data for display on the grey-scale recorder. We shall discuss this processing in more detail in section 4 . As seen in the timing diagram (Figure 3), data processing must be completed before the next $A / D$ conversion sequence begins. The amount of time avail- 


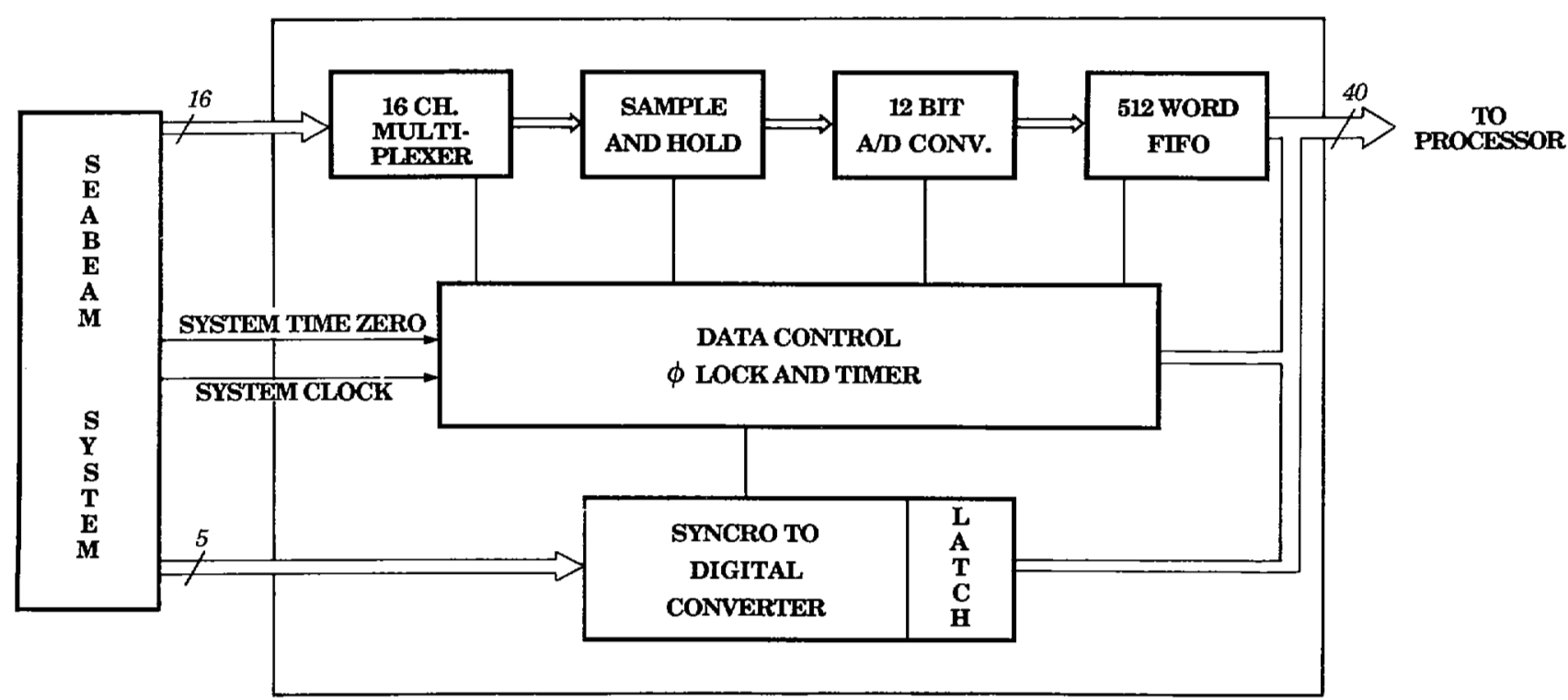

Figure 2: Interface architecture

SONAR TIME ZERO -

DEILAY COUNTER $\sqrt{\text { DEPTH DEPENDENT }}$

A/D CONVERTING

STD CONVERTING

FTFO LEVEL

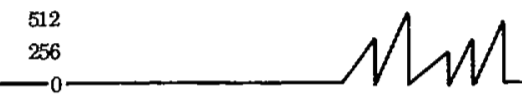

RECORDER TIME ZERO

$几$

$几$

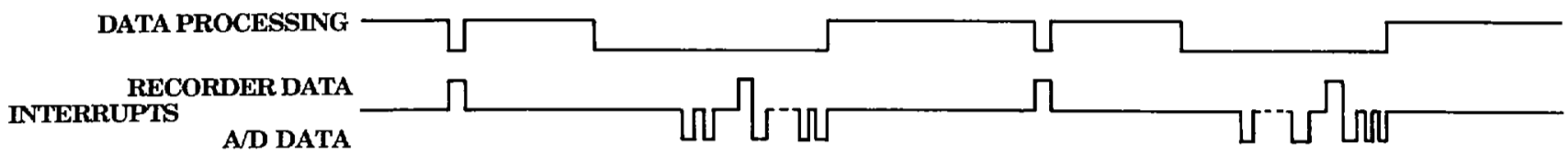

Figure 3: Timing diagram showing the sequence of events happening between two sonar transmission cycles.

able for processing is therefore dependent on the water depth which determines the sonar key pulse interval (e.g. $6 \mathrm{~s}$ in $3000 \mathrm{~m}$ of water depth) and on the number of samples required for each channel (e.g. 500 sanıples in $3000 \mathrm{~m}$ of water depth).

During all the above operations, the recorder sends interrupts to the processor at a rate determined by the scan rate selected by the operator (typically 1-4 s). However, these recorder interrupts are given a lower priority level than the A/D interrupts so that the processor does not service them while A/D interrupts are pending.

\section{DATA PROCESSING}

When the required number of samples of echo amplitudes and roll information have been digitized and stored in a data buffer in memory, the microprocessor stops all digitization and begins processing the data. In the current configuration, three main processing tasks are performed: bottom traking, roll correction and data formatting.

Bottom tracking consists in finding the first amplitude exceeding a given threshold in any one of the 16 preformed beams. The position of this data point with respect to the beginning of the data buffer gives the time elapsed since the onset of the digitization 
sequence to receive the first bottom echo. The bottom tracking routine strives to optimize this time interval and to constrain it within a $200 \mathrm{~ms}$ window by adjusting the time delay between the sonar key pulse and the onset of digitization (Figure 3). Because noise spikes and interferences from other sound sources operating simultaneously with the Sea Beam system occasionally cause false bottom detection, bottom tracking history from previous transmission cycles is also included in the computation of this time delay adjustment.

The first arrival found by the bottom tracking routine is then used as a time reference to compute a port and a starboard time series of echo amplitudes. However, in order to determine which beams belong to either port or starboard a roll correction must be applied. The preformed beams are nominally spaced $22 / 3^{\circ}$ apart and centered in the ship's reference frame as shown in Figure 4 . This figure is an example of bottom echo amplitudes received and detected on the 16 preformed beams for one sonar transmission cycle. The $\mathrm{x}$-axis is time in seconds after transmission, and amplitudes are displayed in volts and have been corrected for acoustic transmission loss through the water column by a time-varying gain in the Sea Beam echo processor hardware. The digitized roll angles correspond to the angle between the ship's vertical axis and true vertical during echo reception. As beams are equally spaced, these roll angles can be used to enter a table of beam offsets with respect to vertical and the roll correction is performed through a table look-up. Note that this roll correction scheme is only a first approximation which is sufficient to produce a useable time series of amplitudes as a function of slant range. For a display of amplitudes as a function of horizontal range, the roll correction needs to take ray-bending effects into account.

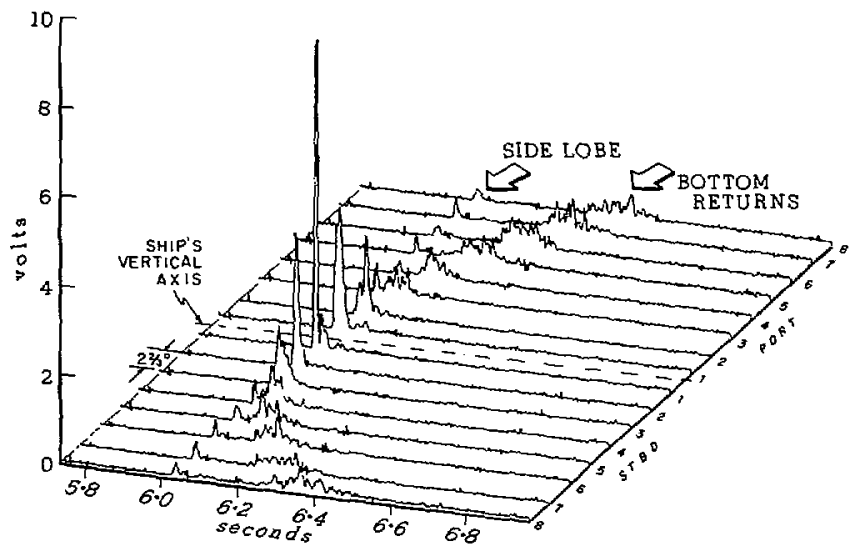

Figure 4: Envelopes of bottom echoes received by the 16 preformed beams.

In the present configuration, the time series on both port and startboard are formed through a peak detection process at incremental slant ranges from the first arrival. These data are then converted from the 12-bit format given by the $\mathrm{A} / \mathrm{D}$ conversion to a 4bit format by table look-up. This yields 16 levels of grey to be displayed on the recorder. The grey-level conversion table can be modified to accomodate overall differences in signal amplitude levels from one Sea Beam system to another. Although not currently implemented, provisions have also been made to include a table look-up correction for angular dependence of seafloor acoustic backscatter.

\section{RESULTS}

\section{A. Seafloor Acoustic Images}

An example of the acoustic image resulting from the above pro- cessing is shown in Figure 5. This image (Figure 5a) was actually replayed from Sea Beam acoustic data recorded aboard the R.V.Thomas Washington on the East Pacific Rise and processed as described in section 4. The cross-track dimension in seconds corresponds to differential slant range (seconds from first arrival). The along-track dimension of this image is in kilometers and matches that of the corresponding swath contour plot produced by the Sea Beam system (Figure $5 \mathrm{~b}$ ). The contour intervals are $10 \mathrm{~m}$ in the upper portion of the swath (1) and $20 \mathrm{~m}$ in the lower portion (2). The acoustic image and the contoured swath complement each other as the qualitative textural information seen in the image would be missed if one only had the contours. Likewise, the quantitative bathymetry given by the contours could not be inferred reliably from the acoustic image. The fact that the lineation pattern seen in the acoustic image is not obvious in the contours at the 10 $\mathrm{m}$ or $20 \mathrm{~m}$ contour intervals indicates that the corresponding relief is lower than $10 \mathrm{~m}$. Although bathymetry data from the Sea Beam
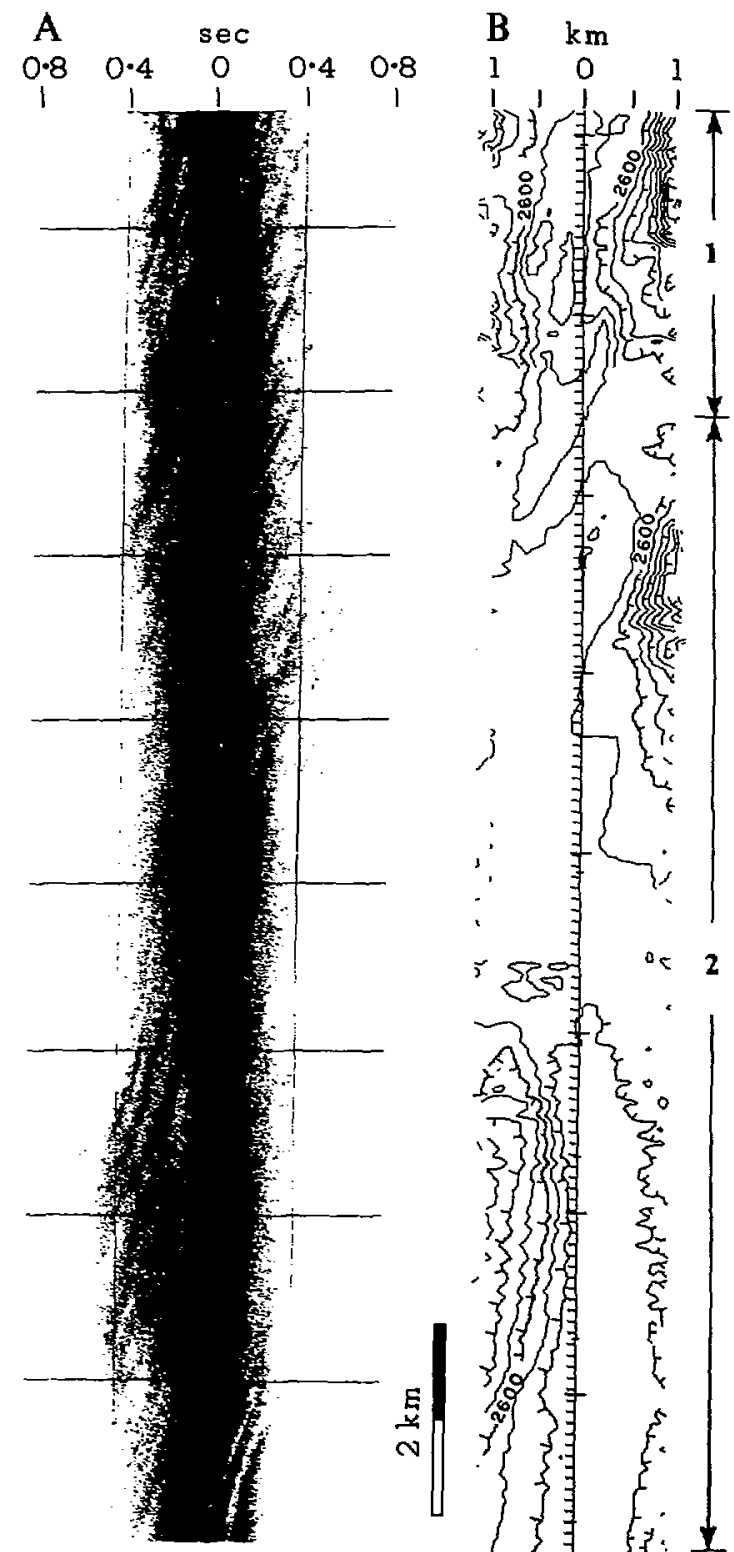

Figure 5: Sea Beam acoustic image of the seafloor. (A) and corresponding contoured bathymetry (B). 
system can be contoured at intervals lower than $10 \mathrm{~m}$, such contours are typically noisy and are therefore difficult to interpret. Note also that the trend of the lineations seen in the acoustic image is distorted by the slant-range representation, the actual trend would appear at a greater angle to the ship's track.

\section{B. Images of the Deep Scattering Layers}

A relatively simpler form of on-line Sea Beam acoustic display consists in outputting the echo amplitude as a function of time for individual beams. This type of display is useful as a quick check of the performance of the echo-sounder part of the Sea Beam system. It is also most useful when tracking Deep Scattering Layers (DSL) in the ocean. Figure 6 is an example of such an on-line acoustic image of the DSL recorded at dusk in the tropical Northern Pacific aboard the R.V. Atlantis II. Three individual beams are represented in this picture (port 8, port 1 and starboard 7) corresponding to incidence angles of roughly $20^{\circ}, 2^{\circ}$ and $-18^{\circ}$ respectively. The horizontal axis spans approximately $60 \mathrm{nmi}$ and the vertical lines are spaced $30 \mathrm{~min}$ apart with the ship progressing at 12 knots from left to right. In this on-line display, spacing between the $30 \mathrm{~min}$ time marks varies as a function of the transmission repetition rate which is determined by the water depth. Closer time marks indicate a deeper bottom. The vertical axis is slant range in uncorrected meters $(1500 \mathrm{~m} / \mathrm{s})$. This slant range is equivalent to depth for the center beam (port 1). The pattern seen in all three beams of Figure 6 is the well known migration pattern of midwater nekton towards the sea surface at night-fall $(7,8)$. On the left side of the image, discrete layers converge upward to the $2 \mathrm{~h}$ time mark and some of the scatterers seem to reassemble into discrete layers in the first $150 \mathrm{~m}$ towards the right of the image while layers are still visible between $400 \mathrm{~m}$ and $600 \mathrm{~m}$.

Compared with similar images of the DSL obtained in the past with conventional wide-beam $12 \mathrm{kHz}$ echo-sounders these images offer greater spatial resolution both along and across the ship's track. The $1622 / 3^{\circ}$ beams of the Sea Beam system yield 16 simultaneous discrete measurements where the conventional echo-sounder integrates over the same volume of ocean to give one measurement. This higher spatial resolution makes it possible to investigate the patchiness of the distribution of the $12 \mathrm{kHz}$ biological scatterers in the DSL's and to provide real-time clues on the lateral extent of a given patch during trawling operations.

\section{CONCLUSIONS}

The on-line Sea Beam acoustic imaging system we have described in this paper allows investigators to extract more information out of the echo signals received by the Sea Beam system than is available in the standard swath bathymetry output. We have shown that acoustic images of the bottom or of the DSL's can be derived from the echoes received on the 16 preformed beams with a small amount of hardware and without interfering with the normal bathymetric function of the Sea Beam system. The additional information provided by this imaging system is a real-time complement to the contoured bottom data and it allows the investigator to make better decisions about survey patterns and data quality while at sea.

Future improvements to this imaging system should include a capability to incorporate the bathymetric information produced by the Sea Beam echo processor into the data processing software in order to perform slant-range correction on seafloor acoustic images. The depths and cross-track distances computed for each transmission cycle could be input on the microprocessor bus through a parallel port. Such a data transmission scheme as already been implemented on the microprocessor as we use it as a spooling interface between our LSI-11/73 minicomputer and the linescan recorder for post-processing operations and for play backs from digitally taperecorded data.

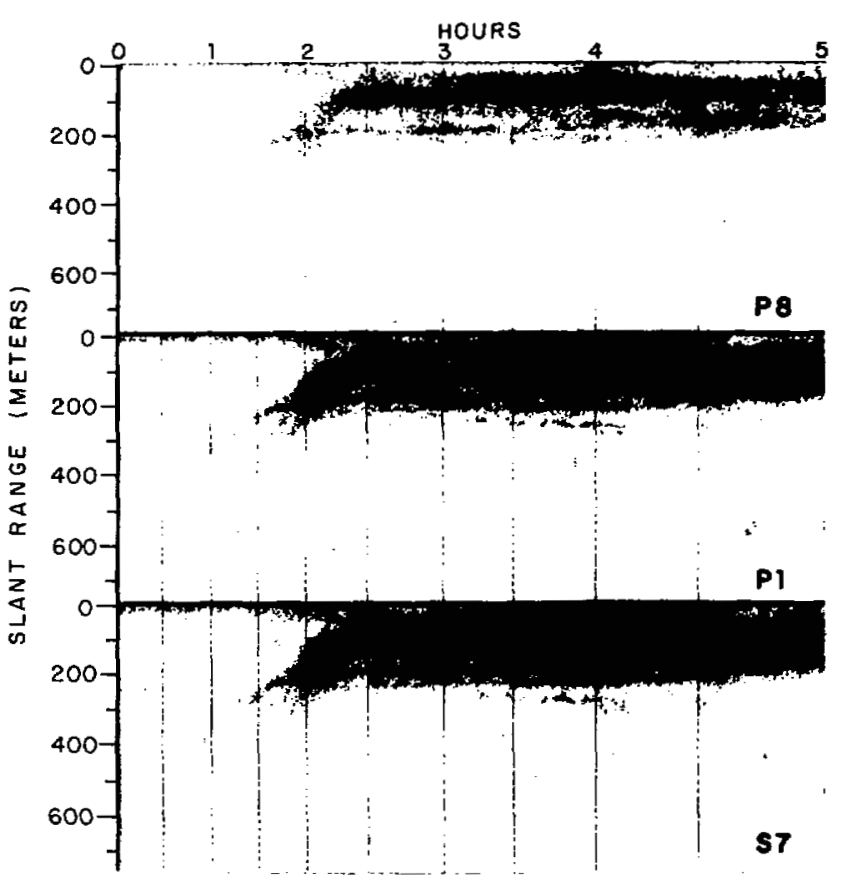

Figure 6: Tracking the DSL on individual beams.

\section{ACKNOWLEDGEMENTS}

The work reported here has been supported by the Office of Naval Research (contract No N00014-79-C-0472) whose funding is gratefully acknowledged.

\section{REFERENCES}

1. de Moustier, C., 1985, Sea Beam acoustic data acquisition system, Marine Physical Laboratory, Scripps Institution of Oceanography, San Diego CA.

2. de Moustier, C. and F.V. Pavlicek, 1987, A fully transportable Sea Beam complex acoustic data acquistion system, OTC 5514, Proc. 19th Offshore Tech. Conf., Vol. 3, pp. 269-274.

3. Renard V. and J.P. Allenou, 1979, Sea Beam Multibeam echosounding in Jean Charcot. Description, evaluation and first results, Int. Hydrog. Rev., Vol. 56(1), pp. 35-67.

4. Farr, H.K., 1980, Multibeam bathymetric sonar: Sea Beam and Hydrochart, Mar. Geol., Vol. 4, pp. 77-93.

5. de Moustier, C. and M.C. Kleinrock, 1986, Bathymetric artifacts in Sea Beam data: how to recognize them and what causes them, J.G.R., Vol. 91, No. B3, pp. 3407-3424.

6. de Moustier, C., 1986, Approaches to acoustic backscattering measurements from the deep seafloor, in Current Practices and New Technology in Ocean Engineering, OED-Vol. 11, A.S.M.E., T. McGuinness and H.H. Shih eds., pp. 137-143.

7. Farquhar, G.B., 1977, Biological sound scattering in the oceans: a review, in Oceanic sound scattering prediction, Plenum Press, N.R. Andersen and B.J. Zahuranec eds., pp. 493-527.

8. Raitt, R.W., 1948, Sound scatterers in the sea, J. Marine Res., Vol VII, No. 3, pp. 393-409. 